\title{
Local Laser Annealing in Exchange-Biased Films with Out-of-Plane and In-Plane Magnetic Anisotropy
}

\author{
S. D. Choi ${ }^{1}$, S. W. Kim² ${ }^{2}$ D. H. Jin ${ }^{1}$, D. K. Yun ${ }^{1}$, M. S. Lee ${ }^{1}$, J. H. Ahn ${ }^{1}$, \\ H. W. Joo' ${ }^{1}$ K. A. Lee ${ }^{1}$, S. S. Lee ${ }^{2}$, and D. G. Hwang ${ }^{2 *}$ \\ ${ }^{1}$ Department of Physics, Dankook University, Cheonan 330-714, Korea \\ ${ }^{2}$ Department of Computer and Electronic Physics, Sangi University, Kangwon 220-702, Korea
}

(Received 6 March 2006)

\begin{abstract}
Local magnetization reversal in the exchange-biased $\mathrm{NiFe} / \mathrm{FeMn}$ and $[\mathrm{Pd} / \mathrm{Co}]_{5} / \mathrm{FeMn}$ multilayers with in-plane and out-of-plane magnetic anisotropy was achieved by using laser annealing. The local annealed $\mathrm{NiFe} / \mathrm{FeMn}$ film under the opposite magnetic field shows a magnetoresistance (MR) curve having two symmetric peaks at the positive and negative exchange biasing field $\left( \pm H_{e x}\right)$. The intensity of the nucleated MR peak rises as the exposed area extends during the laser annealing process, and the peak disappears under the reverse magnetic field. In the case of $[\mathrm{Pd} / \mathrm{Co}] / \mathrm{FeMn}$ films, the local magnetization reversal increased gradually as the laser power increases. The locally reversed magnetization was restored under the opposite magnetic field.
\end{abstract}

Key words : laser annealing, exchange biasing, local magnetization reversal, perpendicular anisotropy

\section{Introduction}

To improve a technical difficulty and simplify the design of a memory device, the current controlled magnetization reversal had been suggested due to the movement of the domain wall and spin transfer in magnetic thin films [1]. An external magnetic field and current can control the magnetization reversal of a free ferromagnetic (FM) element. However, that of the FM element pinned by antiferromagnet (AF) should be realized through thermal annealing for the whole films, and it is hard to get local magnetization reversal. The control of local magnetization reversal of the pinned FM element can be applied to the magnetic memory device and sensors [2].

Exchange biasing at $\mathrm{FM} / \mathrm{AF}$ thin film interfaces has been of interest to fundamental research. Several models focused on the domain structure of the AF layer for compensated and uncompensated interfaces assuming single domain state or uniform magnetization of the FM layers [3, 4]. Although these theories can explain the magnitude of biasing field, they fail to account for the FM domain structure during magnetization reversal and the origin of exchange biasing in the films with perpendi-

*Corresponding author: Tel: +82-33-730-0413,

Fax: +82-33-730-0403, e-mail: dghwang@sangji.ac.kr cular magnetic anisotropy.

In these works, the local magnetization reversal of the exchange-biased FeMn/NiFe bilayers and $[\mathrm{Pd} / \mathrm{Co}]_{5} / \mathrm{FeMn}$ multilayers with an in-plane and out-of-plane magnetic anisotropy was investigated by using laser annealing as a laser power, and according to the exposed area and the direction of the external magnetic field.

\section{Experiments}

$\mathrm{Ta}(5 \mathrm{~nm}) / \mathrm{NiFe}(11 \mathrm{~nm}) / \mathrm{FeMn}(16 \mathrm{~nm}) / \mathrm{Ta}(5 \mathrm{~nm})$ and $\mathrm{Ta}(5 \mathrm{~nm}) /[\operatorname{Pd}(0.8 \mathrm{~nm}) / \mathrm{Co}(0.8 \mathrm{~nm})] \times 5 / \mathrm{FeMn}(15 \mathrm{~nm}) /$ $\mathrm{Ta}(5 \mathrm{~nm})$ multilayers were deposited using ion-beam and dc magnetron sputtering system with a base pressure of $6.0 \times 10^{-9}$ and $1.0 \times 10^{-7}$ Torr, respectively. The strip patterned films of $1 \mathrm{~mm} \times 15 \mathrm{~mm}$ were exposed by the emission of diode pumped solid state (DPSS, Nd:YAG) laser operating at a wavelength of $532 \mathrm{~nm}$ and having a continuous wave $(\mathrm{CW})$ second harmonic generation (SHG) output in 15 minutes under the applied field $\left(\boldsymbol{H}_{\boldsymbol{a}}\right)$ of 600 gauss. The laser beam is focused on a $1 \mathrm{~mm}$ circular spot through an optical fiber its intensity was increased up to $350 \mathrm{~mW}$. During laser annealing, the $\boldsymbol{H}_{\boldsymbol{a}}$ was applied to the opposite direction of the deposition magnetic field $\left(\boldsymbol{H}_{\boldsymbol{d}}\right)$. The hysteresis loops for the exchange-biased films were analyzed by the anisotropy 
magnetoresistance (MR) curve and the magneto-optical Kerr effect (MOKE), and the Extraordinary Hall effect (EHE).

\section{Results and Discussion}

We prepared $\mathrm{Ta}(5 \mathrm{~nm}) / \mathrm{NiFe}(11 \mathrm{~nm}) / \mathrm{FeMn}(16 \mathrm{~nm}) / \mathrm{Ta}(5$ $\mathrm{nm})$ and $\mathrm{Ta}(5 \mathrm{~nm}) /[\mathrm{Pd}(0.8 \mathrm{~nm}) / \mathrm{Co}(0.8 \mathrm{~nm})] \times 5 / \mathrm{FeMn}(15$ $\mathrm{nm}) / \mathrm{Ta}(5 \mathrm{~nm})$ multilayers in order to compare magnetization reversal dynamics of the films with in-plane anisotropy and out-of-plane anisotropy.

Fig. 1 shows the MR curves of $\mathrm{NiFe}(11 \mathrm{~nm}) / \mathrm{FeMn}(16$ $\mathrm{nm}$ ) bilayers as a function of laser power. The MR peak of as-deposited sample before annealing was generated at $+87 \mathrm{Oe}$, and its intensity is $0.9 \%$. After laser annealing, the new MR peak was nucleated at $-63 \mathrm{Oe}$, and its region was shifted to $-80 \mathrm{Oe}$ as the power increases to $400 \mathrm{~mW}$. Also its intensity is increased from $0.1 \%$ at $200 \mathrm{~mW}$ to $0.3 \%$ at $400 \mathrm{~mW}$. The original MR peak disappeared through laser annealing of $400 \mathrm{~mW}$ in 15 minutes. The nucleation and annihilation of MR peaks are due to local reversal of exchange biasing in $\mathrm{NiFe} / \mathrm{FeMn}$ bilayers. The nucleated MR peak was gradually increased as the exposed area enlarges into sample. Therefore, the local reversal of exchange anisotropy could be controlled by laser annealing process.

To restore the direction of exchange anisotropy, the laser annealing of sample 2 annealed at $400 \mathrm{~mW}$ under reverse magnetic field was investigated, as shown in

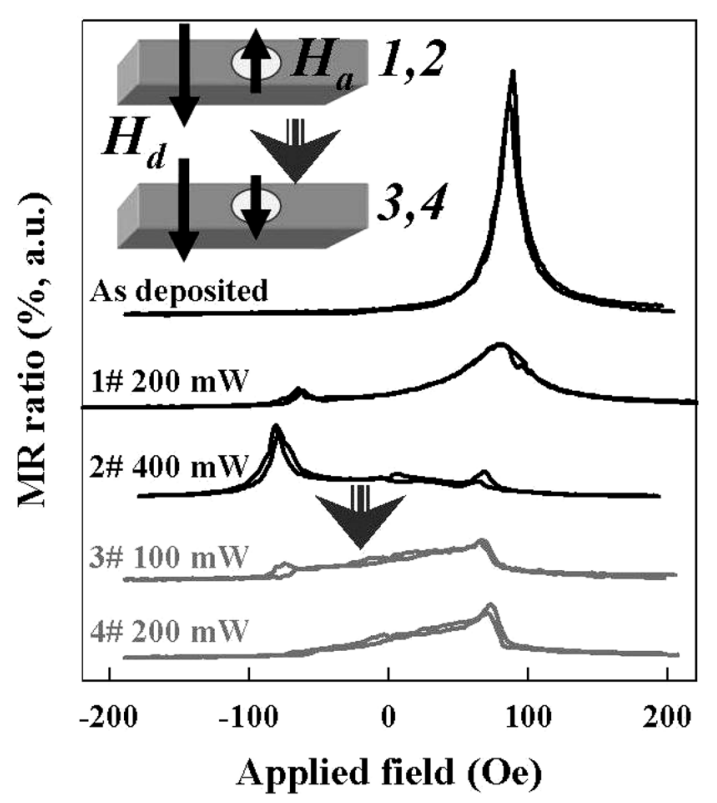

Fig. 1. The change of MR curves of the strip-patterned $\mathrm{NiFe}(11 \mathrm{~nm}) / \mathrm{FeMn}(16 \mathrm{~nm})$ bilayers as a function of laser power and the direction of applied field.

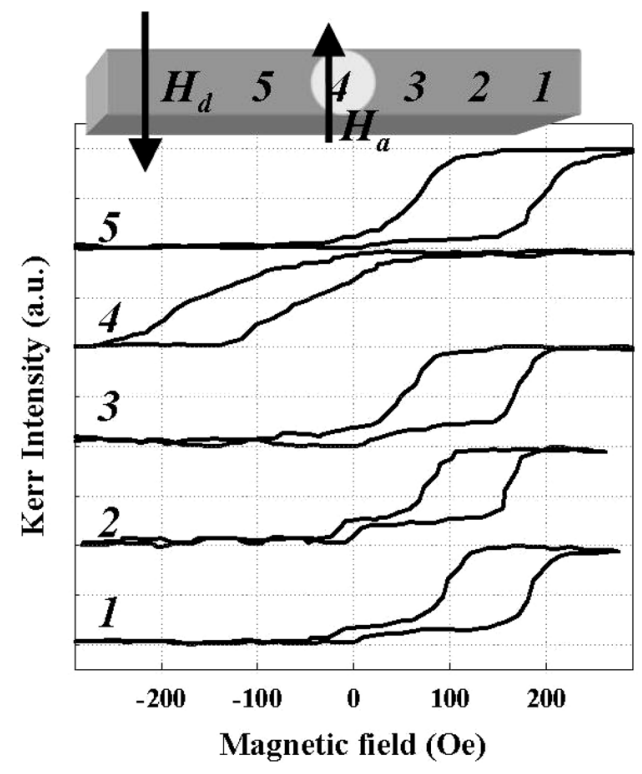

Fig. 2. The MOKE curves of the $2 \mathrm{~mm} \times 10 \mathrm{~mm}$ strip-patterned $\mathrm{NiFe}(10 \mathrm{~nm}) / \mathrm{FeMn}(10 \mathrm{~nm})$ bilayers changing from right to left, where the size of the MOKE spot is $2 \times 2 \mathrm{~mm}$, and the laser spot is at region 4.

figure 1 (see samples 3 and 4). The MR peak nucleated at -80 Oe was vanished at the sample 4 of $200 \mathrm{~mW}$, and the original peak was regenerated again at +80 Oe. However, its intensity was shrunk by the damage of laser spot and intermixing between $\mathrm{NiFe}$ and FeMn. Therefore, the laser power has to be reduced to avoid the damage of laser.

Fig. 2 shows the MOKE curves of patterned $\mathrm{NiFe}(10$ $\mathrm{nm}) / \mathrm{FeMn}(10 \mathrm{~nm})$ bilayers from the right region of the laser spot to the left one. From the MOKE curves, we can find that the $\boldsymbol{H}_{e x}$ at the outside region of laser spot (region 1, 2, 3 and 5) are between +110 and +125 Oe. However, the $\boldsymbol{H}_{e x}$ of the spot region 4 was reversed to $-100 \mathrm{Oe}$, which proves that the local reversal of exchange anisotropy could be carried out using laser annealing.

Fig. 3 shows (a) the normalized Hall voltage (NHV) and (b) the differential NHV curves of the perpendicular exchange-biased $[\mathrm{Pd} / \mathrm{Co}]_{5} / \mathrm{FeMn}$ multilayers as a function of laser power, where the peaks correspond to the steps of the NHV loops. The $\boldsymbol{H}_{e x}$ shifted from -143 Oe at asdeposited (ASD) sample to +26 Oe at $350 \mathrm{~mW}$. The local reversal phenomena of perpendicular exchange anisotropy differs from that of an in-plane one because the magnetic domain of $[\mathrm{Co} / \mathrm{Pd}]$ multilayers has a very small column structure. The local magnetization reversal in the $\mathrm{NiFe} /$ FeMn film will generate the complex domain walls at the boundary to reduce demagnetization energy, but the reversal in the $[\mathrm{Pd} / \mathrm{Co}]$ film has a simple domain structure. Therefore, the reversed domains in the perpendicular sample 


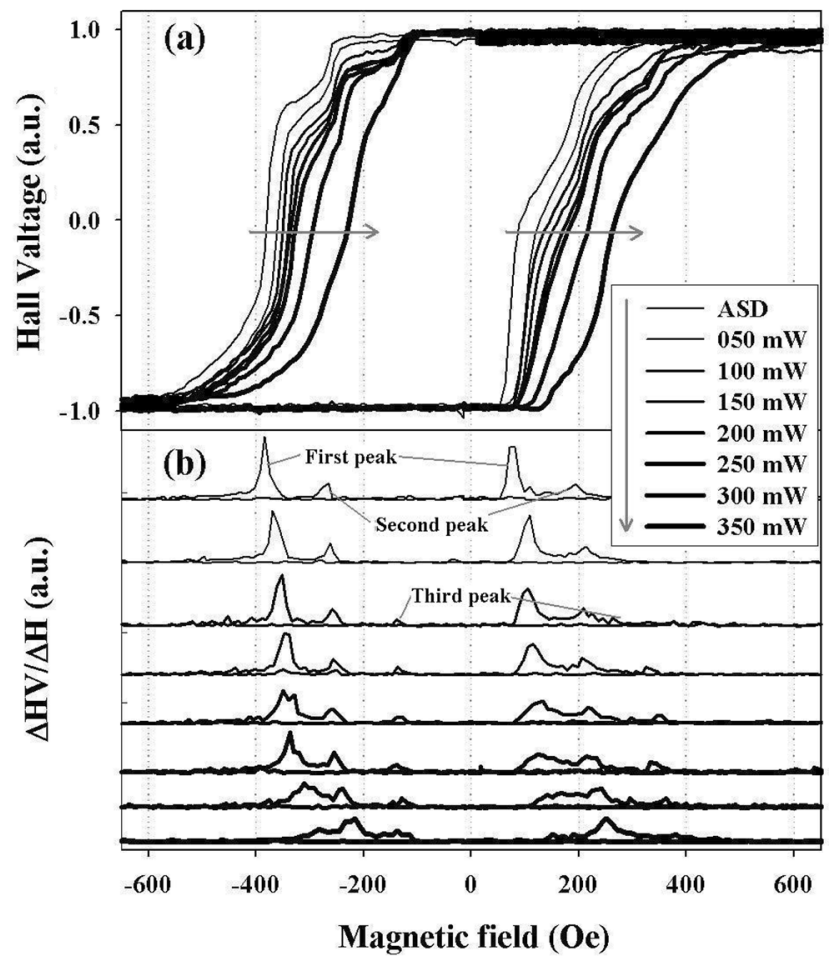

Fig. 3. (a) The NHV hysteresis loops and (b) the differential NHV curves in perpendicular exchange-biased $[\operatorname{Pd}(0.8 \mathrm{~nm}) /$ $\mathrm{Co}(0.8 \mathrm{~nm})]_{5} / \mathrm{FeMn}(15 \mathrm{~nm})$ multilayers as a function of laser $(\mathrm{ASD}=\mathrm{As}$ deposited).

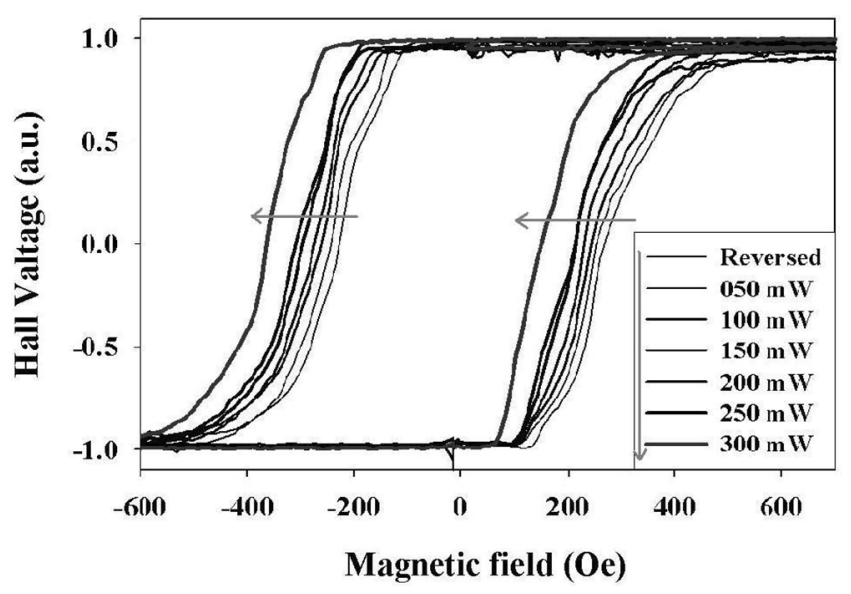

Fig. 4. The $\mathrm{NHV}$ hysteresis loops of $[\operatorname{Pd}(0.8 \mathrm{~nm}) / \mathrm{Co}(0.8$ $\mathrm{nm})]_{5} / \mathrm{FeMn}(15 \mathrm{~nm})$ multilayers as a function of laser power under reversed magnetic field.

will increase gradually as the laser power increases. Therefore, the $\boldsymbol{H}_{\boldsymbol{e} \boldsymbol{x}}$ was shifted according to the direction of the applied field during laser annealing. Figure 4 shows the return process of $\boldsymbol{H}_{e x}$ from +26 Oe to -107 Oe as the laser power increases to $300 \mathrm{~mW}$.

The NHV of as-deposited sample has the asymmetric step at the top left corner. The steps can be found in the

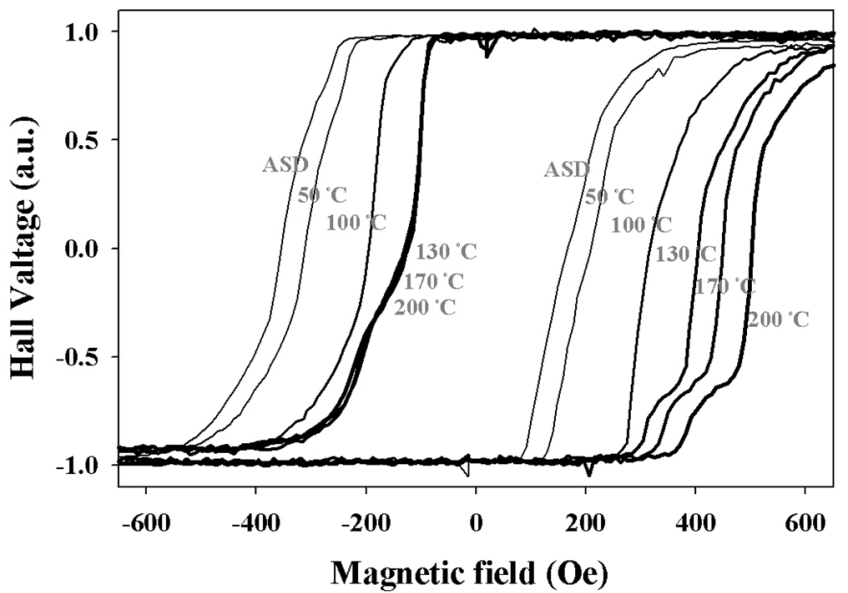

Fig. 5. The NHV hysteresis loops as a function of annealing temperature in $[\operatorname{Pd}(0.8 \mathrm{~nm}) / \mathrm{Co}(0.8 \mathrm{~nm})]_{5} / \mathrm{FeMn}(15 \mathrm{~nm})$ multilayers.

system where the cooling procedure or the growth procedure has been carried out in small fields $[5,6]$. In the case of our samples, the film fabrication was carried out under the stray field below 30 Oe without the external magnetic field. Therefore, the multilayers were not fully saturated magnetic states and the asymmetric steps disappeared according to the increase of the laser power under the external magnetic field of $600 \mathrm{Oe}$, as shown in Figure 3. Also the steps can vanish in the $[\mathrm{Co} / \mathrm{Pd}]$ film without the external magnetic field. When the Co thickness is below $0.4 \mathrm{~nm}$, the hysteresis loop shows a simple square loop. Therefore, in our sample, the $\mathrm{Co}$ and $\mathrm{Pd}$ layers have almost the same thickness at $0.8 \mathrm{~nm}$, and the exchange coupling between Co layers will be weaker than that of thin Co layer.

To compare the relationship between local laser annealing and thermal annealing, the sample reversed at 300 $\mathrm{mW}$ in Figure 4 was annealed thermally up to $200^{\circ} \mathrm{C}$, as shown in Figure 5. The $\boldsymbol{H}_{\boldsymbol{e x}}$ was transferred from -95 Oe to +188 Oe in increasing temperature, and its coercivity was almost between 260 Oe and 300 Oe. The asymmetric step was regenerated at $130{ }^{\circ} \mathrm{C}$ due to the weakening of the external magnetic field during thermal annealing. We used an $\mathrm{Nd}$ magnet of $1 \mathrm{kOe}$ during the thermal process. The $\mathrm{Nd}$ magnet has a low Curie temperature, and its intensity will be reduced rapidly above $100^{\circ} \mathrm{C}[7,8]$.

\section{Conclusion}

Local magnetization reversal of exchange biasing of $\mathrm{NiFe} / \mathrm{FeMn}$ and $[\mathrm{Pd} / \mathrm{Co}]_{5} / \mathrm{FeMn}$ films with in-plane and out-of plane magnetic anisotropy was achieved by laser annealing. The MOKE curves evidenced local magneti- 
zation reversal of $\mathrm{NiFe} / \mathrm{FeMn}$ film with in-plane anisotropy. The magnetization reversal of perpendicular exchange-biased film can be controlled continuously in the air using laser annealing. The asymmetric step in the perpendicular film is due to the unsaturated magnetic states.

\section{Acknowledgement}

This work was accomplished with support from KOSEF No. R01-2005-000-11188-0.

\section{Rerefences}

[1] E. B. Myers, D. C. Ralph, J. A. Katine, R. N. Louie, and R. A. Buhrman, Science, 285, 867 (1999).
[2] J. Fassbender, D. Ravelosona, and Y. Samson, J. Phys. D, 37, 179 (2004).

[3] J. Nogués and Ivan K. Schuller, J. Magn. Magn. Mater. 192, 203 (1999).

[4] R. L. Stamps, J. Phys. D, 33, 247 (2000).

[5] H. W. Zhao, W. N. Wang, Y. J. Wang, W. S. Zhan, and J. Q. Xiao, J. Appl. Phys. 91, 6893 (2002).

[6] P. Milteny, M. Gierlings, M. Bamming, U. May, G. Guntherodt, J. Nogués, M. Gruyters, C. Leighton, and Ivan K. Shuller, Appl. Phys. Lett. 75, 2304 (1999).

[7] S. Soeya, T. Imagawa, K. Mitsuoka, and S. Narishige, J. Appl. Phys. 76, 5356 (1994).

[8] H. W. Joo, S. W. Kim, J. H. An, J. H. Choi, M. S. Lee, K. A. Lee, D. G. Hwang, and S. S. Lee, J. of Magnetics 10(1), 33 (2005). 